Tohoku J. exp. Med., 1973, 109, 377-383

\title{
New Test Methods for Estimation of Facial Weakness
}

\author{
Jiro Hozawa, Yoshitaka Sasaki, Shinobu Takahashi \\ and Jun Kusakari \\ Department of Otolaryngology, Tohoku University School of \\ Medicine, Sendai
}

Hozawa, J., Sasaki, Y., Takahashi, S. and Kusakari, J. New Test Methods for Estimation of Facial Weakness. Tohoku J. exp. Med., 1973, 109 (4), 377-383

New methods of electrical recording of facial weakness were proposed which provides means of quantitative estimation of facial weakness. Graphic presentation of the dynamic aspects of facial weakness was made possible by recording of interlabial pressure through a pressure transducer during whistle action, recording of resistance changes between two electrodes produced by wrinkle and smile actions, and by recording of blinking speed with an electronystagmography. These tests are simple, quantitative, and applicable to patients without discomfort. Differences between healthy and affected sides in these records provide reliable information on the effect of treatment. A simultaneous recording of these graphs is valuable to demonstrate associated movements. —— brain nerve; ear

Accurate assessment of therapeutic effect on facial palsy cannot be made without objective evaluation of facial weakness, for which photography is the most popular method. However, the photography provides neither dynamic aspect nor quantitative measure of facial weakness. Facial muscle weakness can be analyzed by an electromyography which also furnishes the important prognostic information (Wigand, 1967; Wigand et al. 1968; Hiestond et al. 1969; Totsuka, et al. 1970; Yanagihara and Kishimoto 1972). But, the actual state of facial asymmetry cannot be visualized by the electromyography.

In order to measure the grade of facial weakness, the authors have devised the new quantitative tests, namely, the whistle test, the smile test, the wrinkle test, and the blink test. The method of these tests and some of the results are presented in this paper.

\section{Methods of Test and Results}

\section{The Whistle Test (Labial Dynamography)}

The blockdiagram in Fig. 1 presents the principle of this test. While the patients whistles, the interlabial pressures of normal and affected sides are transmitted into two gum-caps in the patients mouth. The pressure in each gumcap is converted into an electrical signal by a transduser. The signal is transmitt-

Received for publication, August 21, 1972.

Reprints request to the Department of Otolaryngology, Tohoku University School of Medicine, Seiryomachi, Sendai, Japan. 


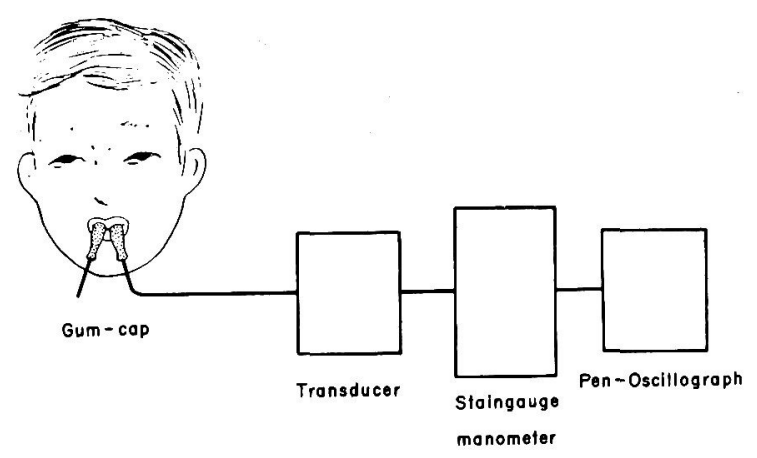

Fig. 1. Principle of whistle test.

When the patient whistles, labial pressure is produced by a contraction of the orbicular oculi muscle and compress a gum-cap in the patient's mouth. As the gum-cap is connected with a transducer, the labial pressure is amplified by the straingauge manometer, and is recorded in the pen-oscillograph.

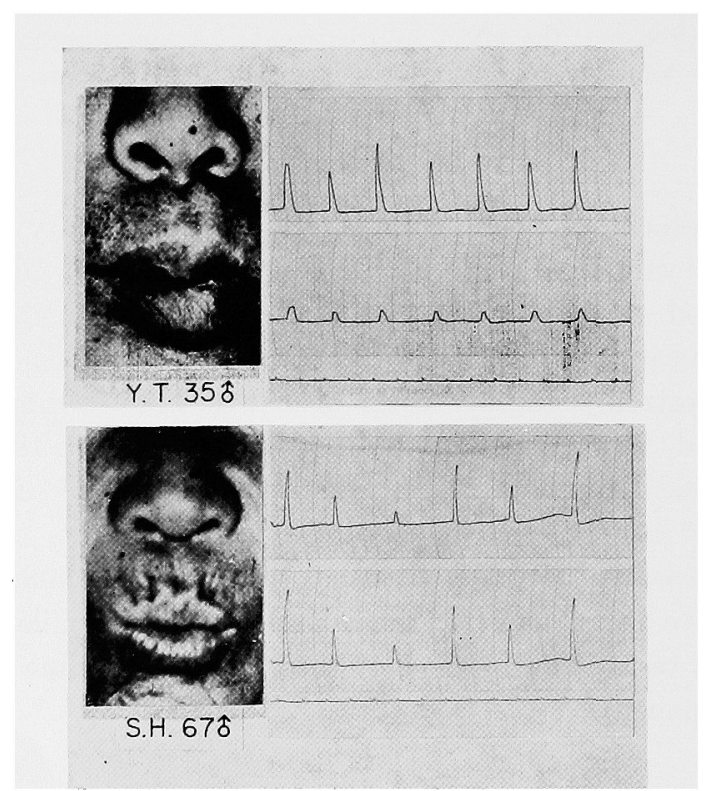

Fig. 2. Representative tracings of labial dynamography comparing with whistle action. The upper case is traumatic facial palsy. The side-difference (left and right) in labial asymmetry was remarkable. The lower case shows a stage of restoration of Bell's palsy. The side-difference in labial pressure disappeared in accordance with the diminution of labial asymmetry.

ed into a strain-gaugemanomter, amplified, and fed into one of the channel of a two chanells pen-oscillograph. The authors named this method the labial dinamography.

Fig. 2 is a couple of examples to show the good accordance of labial symmetry with the interlabial pressure. Labial asymmetry by a traumatic facial palsy is 


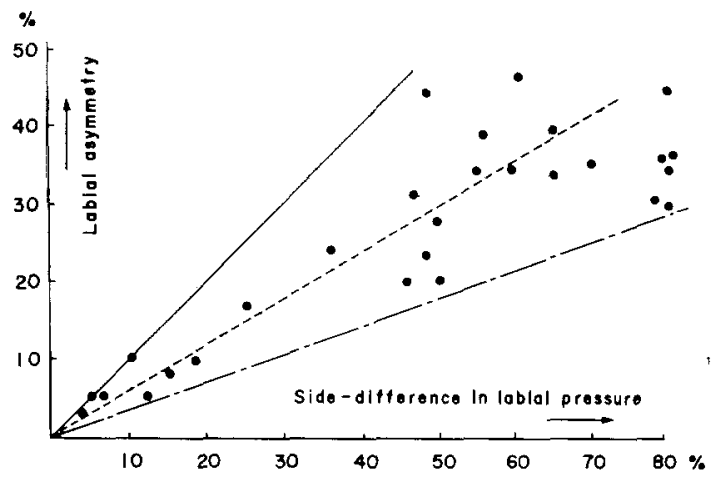

Fig. 3. Relationship between labial asymmetry and labial dynamography in 28 cases. The ordinate shows the degree of labial asymmetry and the abscissa shows the sidedifference in labial pressure. The side-difference in labial pressure increased directly according to the increase in degree of labial asymmetry. It was noticeable that, all points on this graph were under the $45^{\circ}$ line.

given in the upper section and labial symmetry by a restorted Bell's palsy is shown in the lower section with corresponding labial dynamography.

Fig. 3 presents a result of quantitative measurements on 28 cases with facial palsy. In the figure, the degree of labial asymmetry is given in the ordinate and difference in the interlabial pressure between normal and paralyzed side is shown in the abscissa. It is noticeable that all points on this graph are under the $45^{\circ}$ line. This means that labial weakness can be more discernible by labial dynamography than by visual determination of labial asymmetry. In the reversible Bell's palsies, difference in labial pressure between each side diminished in parallel with improvement of facial movement.

\section{The Wrinkle Test and The Smile Test}

As shown in Figs. 4 and 5, the wrinkle test and the smile test are based on the same principle. Two electrodes are fixed at a constant distance on the patient's

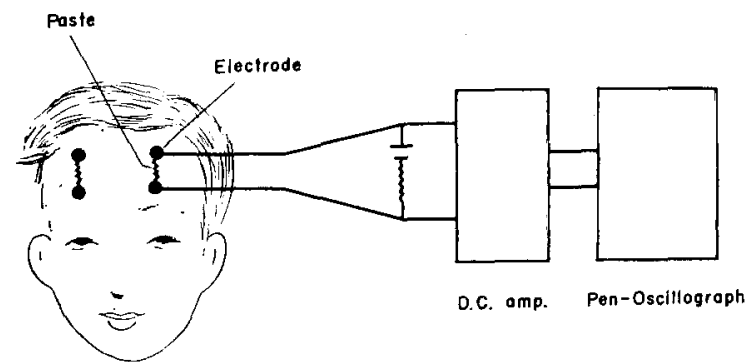

Fig. 4. Principle of wrinkle test.

The two electrodes are fixed on the patients' forehead at a constant interval and connected with each other by paste. Changes in distance between the two electrodes due to wrinkling movement by contraction of the frontalis muscle provoked interelectrode resistance change which is amplified by a D.C. amplifier and fed into a penoscillograph. 


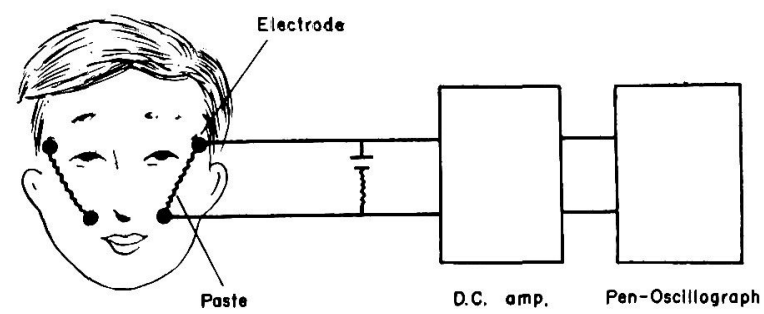

Fig. 5. Principle of smile test.

Two electrodes are fixed between the temple and the corner of the mouth. The two electrodes are bridged with paste. Smiling movement by contraction of the zygomatic muscle is recorded electrically with the same principle as the wrinkle test.

forehead in the wrinkle test, and between the temple and the corner of the mouth in the smile test. The two electrodes are bridged with paste. Changes in distance between the two electrodes due to wrinkling movement by contraction of the frontalis muscle, or smiling movement by contraction of the zygomatic muscle, provoke interelectrode resistance change which is amplified by a D.C. amplifier and fed into a pen-oscillograph.

Figs. 6 and 7 are the examples of the results of these tests comparing with the patient's photographs. In each figure, traumatic facial palsy is presented in the upper section and restoring stage of Bell's palsy in the lower section. In the traumatic case, the degree of paralysis is clearly visualized by a reduced amplitude

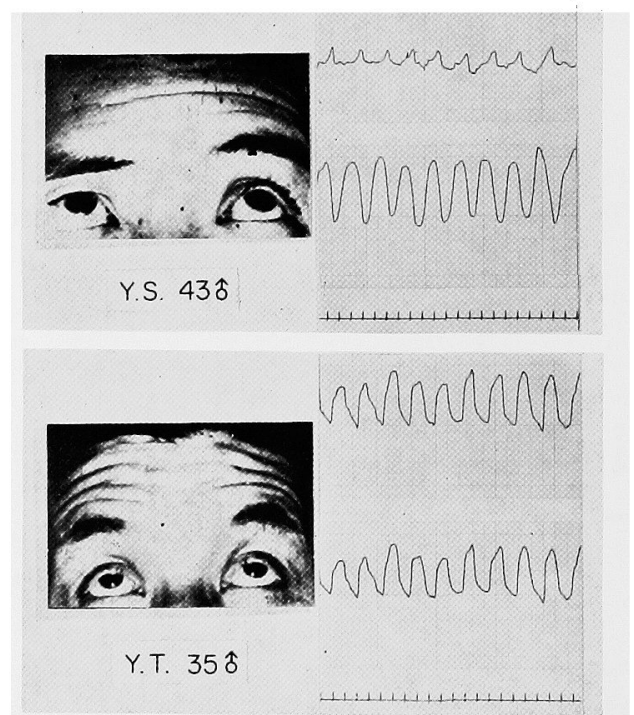

Fig. 6. Representative tracings of wrinkle actions.

The upper case is of traumatic facial palsy. The amplitude of the paralytic side was smaller than that of the normal side. The lower case shows a stage of restoration of Bell's palsy. The side-difference as well as the wrinkle asymmetry was not clear. 


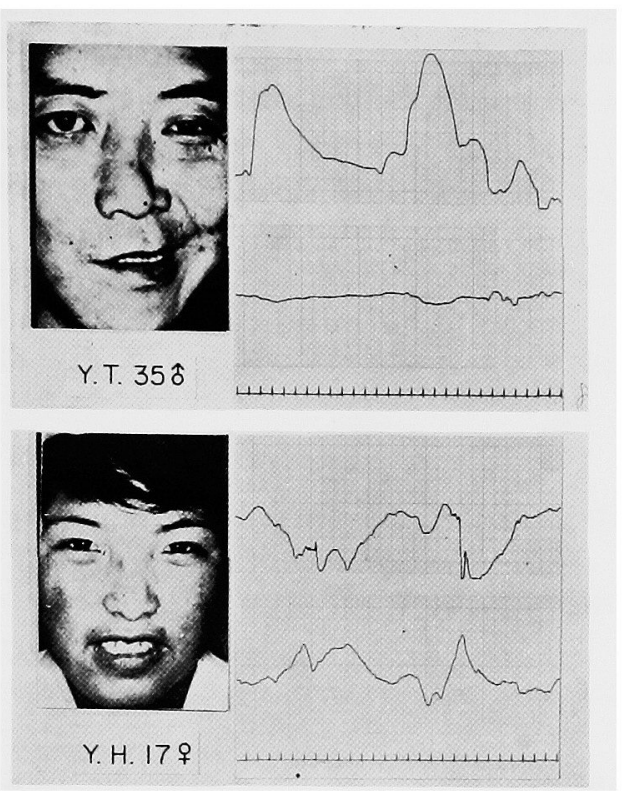

Fig. 7. Representative tracings of smile test comparing with smile actions.

The upper is a case of traumatic facial palsy. Active movement, which showed good contraction of zygomatic muscles, was observed in the sound side, but not in the paralytic side. The lower case shows a stage of restoration of Bell's palsy. In this case, good contraction of the bilateral zygomatic muscles were observed.

of the graph of the involved side, while good contraction of the muscles is evidenced in the case of restoring Bell's palsy.

\section{The Blink Test}

As the blockdiagram in Fig. 8 shows, the blinking speed or speed of vertical eye-movement can be recorded by an electronystagmography. Thus, the delay in the blinking speed on the involved side due to facial muscular weakness can be assessed by comparing with that on the normal side. The blink reflex may be

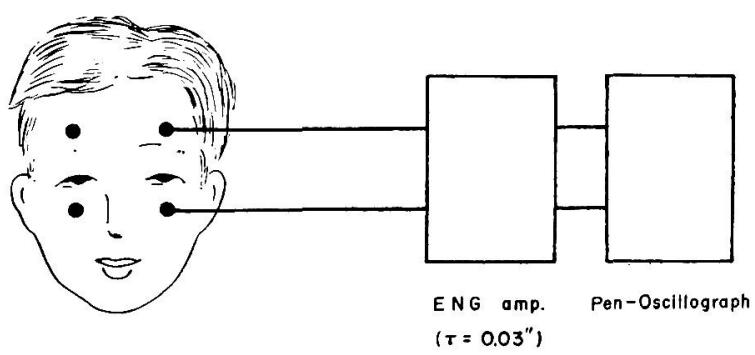

Fig. 8. Principle of blink test.

The speed of vertical eye-movement induced by blinking is recorded by electronystagmography $\left(\tau=0.03^{\prime \prime}\right)$. 
elicited either by a tap on the glabells or by a flash of light. The tap was found to be a more suitable stimulation in our tests. (Hozawa et al. 1966)

Fig. 9 shows the results of the blink test. The upper is a case of taumatic facial palsy in which a lagophthalmos was evident and difference in the blinking speed was remarkable. The lower case shows a stage of restoration of Bell's palsy

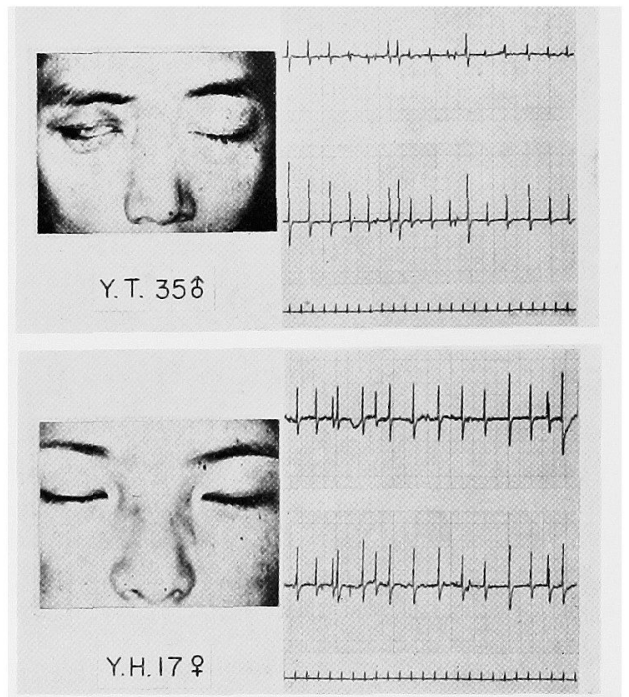

Fig. 9. Representative tracing of blink test comparing with blink actions.

The upper is a case of traumatic facial palsy. In this case, lagophthalmia was clearly found. Also, the side-difference in the blinking speed was remarkable. The lower case shows a stage of restoration of Bell's palsy. In this case, the side-difference in blinking speed as well as lagophthalmia was not clear.

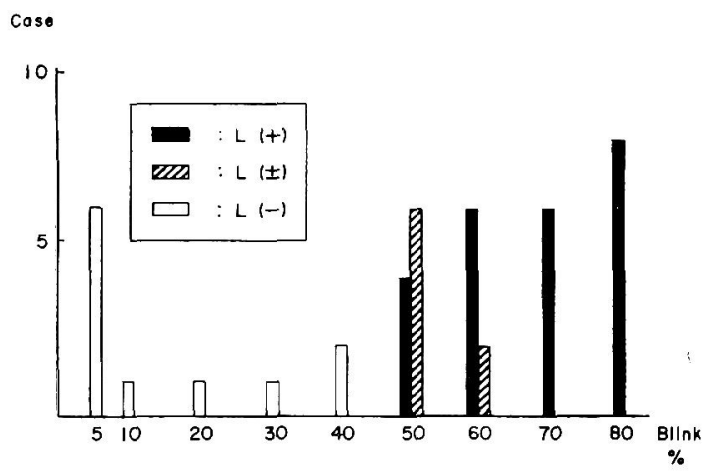

Fig. 10. Relationship between grade of lagophthalmos and the results of blinking test in 43 cases.

The ordinate shows the number of cases and the abscissa shows the side-difference in blinking speed. The side-difference were remarkable in the cases with lagophthalmia $(\mathrm{L}(+))$, but not in the cases without lagophthalmia $(\mathrm{L}(-))$. 
in which difference in blinking speed as well as lagophthalmos was not evident.

Fig. 10 is a result of the blink test in 43 cases of facial palsy. The differences in blinking speed between healthy and affected sides were distinguishable in the cases with lagophthalmos, but not in the cases without lagophthalmos. The normal range of the speed difference was within $5 \%$. It was noticeable that some of the cases without lagophthalmos showed a speed difference higher than $5 \%$. This means that the blink test is a more sensitive method for the detection of facial palsy. As Pulec and House (1964) reported the blink reflex is so sensitive that it is available for the early diagnosis of acoustic tumor.

\section{Conclusion}

The advantages of these tests for recording facial weakness were summarized as follows:

1) These tests are simple and applicable to the patients without any mental or physical discomfort.

2) The degree of paralysis can be determined quantitatively by comparing the records of both sides.

3) Objective evaluation of the treatment can be made accurately by these tests.

4) A polygraph of these tests can be used for the observation of associated movements.

\section{References}

1) Hiestond, P., Kaeser, H., Kocher, R. \& Pfaltz, C.R. (1969) EMG und Facialis Dekompression bei Bell'scner Lämung. Pract. oto-rhinolaryng. (Basel), 31, 144-153.

2) Hozawa, J., Sato, K., Wakayama, R., Sasaki, Y., Ishikawa, K., Arai, E. \& Watanabe, A. (1966) Diagnosis of facial palsy by moans of recording blink-reflex. Otolaryngology (Tokyo) (Jap.), 38, 11-18.

3) Pulec, J.L. \& House, W.F. (1964) Special tests in tha early diagnosis of acoustic neuromas. Laryngoscope (St. Louis), 74, 1183-1193.

4) Totsuka, G., Hirose, H., Kobayashi, T. \& Nimi, S. (1970) A clinical and electromyographic study of postoperative facial palsy. Pract. oto-rhinolaryng. (Basel), 32, 17-28.

5) Wigand, M.E. (1967) Die Prognose der idiopathischen (Bellschen) Fazialisparese bei Electromyografischer Indikationsstellung zur Dekompressionsoperation. Z. Laryng. Rhinol., 46, $439-451$.

6) Wigand, M.E., Spreng, M. \& Bumm, P. (1968) Computer-Analyse von FacialisElektromyogrammen. Acta oto-laryng. (Stockh.), 66, 296-304.

7) Yanagihara, N. \& Kishimoto, M. (1972) Electrodiagnosis in facial palsy. Arch. Otolaryng., 95, 376-382. 